\title{
Fotoinhibición: Respuesta fisiológica de los vegetales al estrés por exceso de luz
}

\section{Photoinhibition: Physiological response of plants to high-irradiance stress}
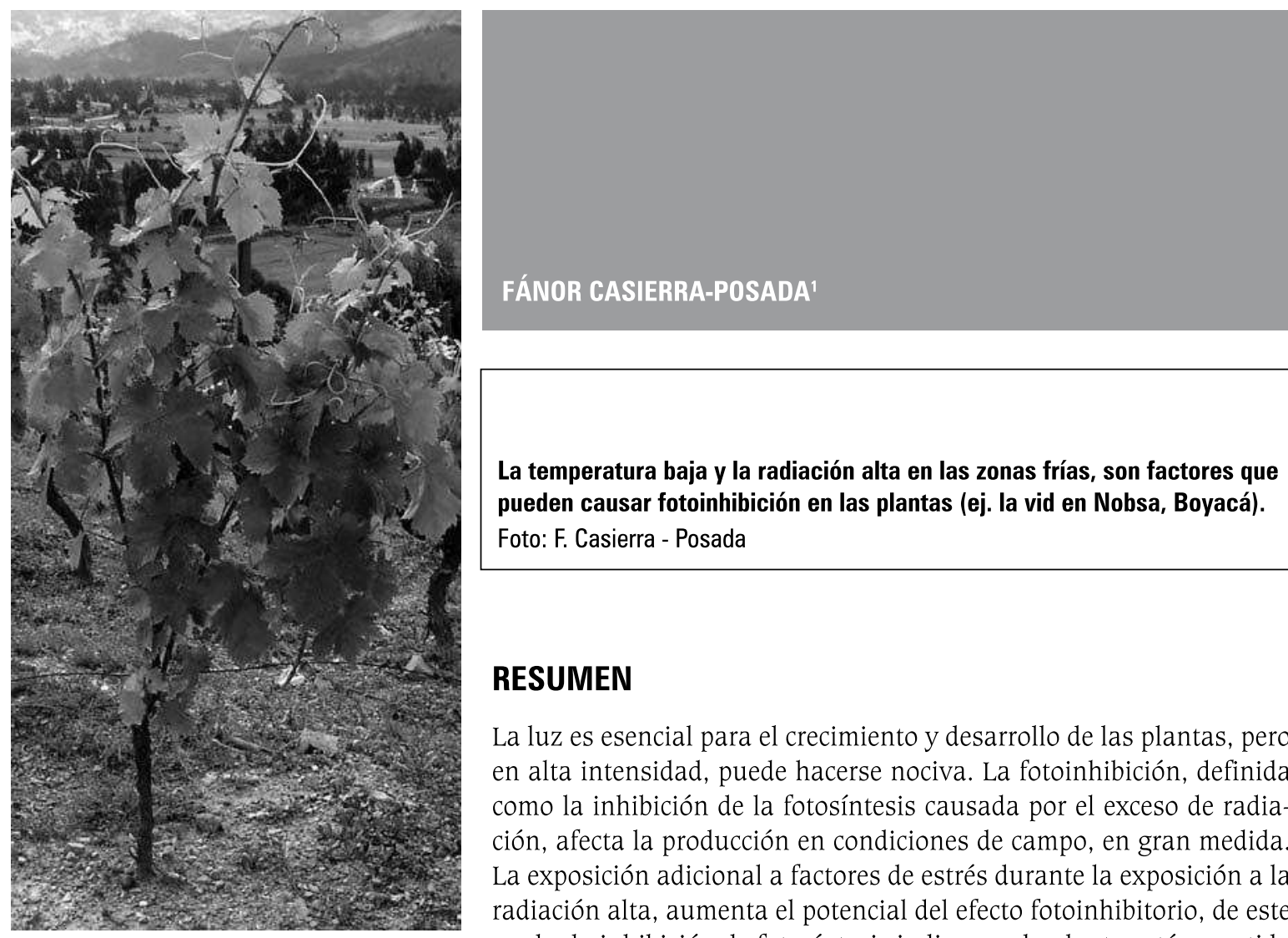

\section{RESUMEN}

La luz es esencial para el crecimiento y desarrollo de las plantas, pero en alta intensidad, puede hacerse nociva. La fotoinhibición, definida como la inhibición de la fotosíntesis causada por el exceso de radiación, afecta la producción en condiciones de campo, en gran medida. La exposición adicional a factores de estrés durante la exposición a la radiación alta, aumenta el potencial del efecto fotoinhibitorio, de este modo, la inhibición de fotosíntesis indica que la planta está sometida a condiciones estresantes. La fotoinhibición puede ser reversible, jugando un papel de protección de los fotosistemas, pero también puede reflejar un daño que ya ha sucedido en el aparato fotosintético, caso en el cual, es irreversible. En esta revisión, se presentan algunos mecanismos fisiológicos y moleculares de la fotoinhibición y la interacción entre la luz y otros factores causantes de estrés y se discuten sus efectos en las plantas.

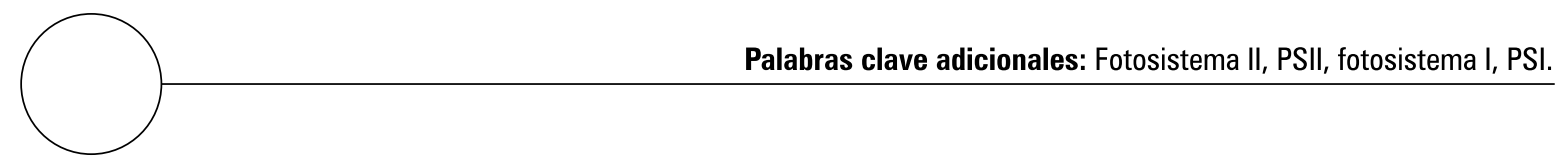

Profesor Asociado, Facultad de Ciencias Agropecuarias, Grupo Ecofisiología Vegetal, Universidad Pedagógica y Tecnológica de Colombia (UPTC), Tunja. fcasierra@tunja.uptc.edu.co 


\section{ABSTRACT}

Light is essential for plant growth and development, but too much of it may be harmful. Photoinhibition, defined as the inhibition of photosynthesis caused by excessive radiance, affects field production at a great extent. The exposure to additional stress factors during exposure to high radiance increases potential for photoinhibitory effects, so that inhibition of photosynthesis indicates that the plant is submitted to stressful conditions. Photoinhibition may be reversible, playing a protective role for the photosynthetic systems, but it can also reflect damage that has already occurred in the photosynthetic apparatus, being irreversible in this case. In this review, some physiological and molecular mechanisms of photoinhibition are presented and the interaction between light and other stress factors and their effects on plants are discussed.

Additional key words: Photosystem II, PSII, photosystem I, PSI.

INTRODUCCIÓN

A pesar de que la luz representa un sustrato fundamental para la realización de la fotosíntesis, una intensidad luminosa que sobrepase el nivel de adaptación de los vegetales, combinada con otros factores causantes de estrés, causa reducción en la actividad fotosintética. Si la energía lumínica absorbida que llega a los centros de reacción, excede la cantidad de energía que puede utilizarse, se pueden generar daños en el aparato fotosintético (Demming-Adams y Adams, 1992; Horton et al., 1994; Lucinski y Jackowski, 2006). El fenómeno de la fotoinhibición se conoce desde hace más de 100 años a través de los trabajos realizados por Ewart (1896) quien se encuentra dentro de los primeros investigadores en estudiar el fenómeno de la fotosíntesis bajo condiciones ambientales reales (Adir et al., 2003). El término fotoinhibición (PI) fue definido originalmente por Kok en 1956, como una reducción en la actividad fotosintética que ocurre como consecuencia de un exceso de iluminación (Yordanov y Veleikova, 2000).
Desde las primeras etapas de la investigación de este fenómeno, se reportó la inactivación de los fotosistemas I y II, es así como se observó la PI del fotosistema I (PSI) en Spinacia oleracea bajo condiciones tanto aeróbicas como anaeróbicas. En estas observaciones, la iluminación de las membranas de los tilacoides con 30.000 lux, bajo condiciones aeróbicas, disminuyó la actividad fotorreductora del $\mathrm{NADP}^{+}$, el cual fue $10 \%$ menos que en el control, y además, se encontró que el $40 \%$ de los centros de sulfuro de hierro (FeS) permanecían intactos (Inoue et al., 1986; 1989). Hasta hace poco, el término fotoinhibición se utilizó principalmente como un sinónimo de PI del fotosistema II (PSII), dado que la proteína D1 había sido considerada como el objetivo principal de la PI (Andersson y Styring, 1991; Barber y Anderson, 1992; Aro et al., 1993), y también, porque el PSI es más estable que el PSI bajo condiciones de iluminación excesiva (Powels, 1984). El daño causado por la luz incluye la inhibición del PSII y la degradación de la proteína D1 (Aro 
et al., 1993). Sin embargo, en la última década, algunas publicaciones muestran que a temperaturas bajas e iluminación escasa, el PSI se ve más afectado que el PSII en algunas plantas (Terashima et al., 1994; Havaux y Davoud, 1994; Sonoike y Terashima, 1994; Tjus et al., 1998; Zak y Pakrasi, 2000).

En relación con la PI en el PSII, en Calathea makoyana, expuesta a temperatura baja $\left(5^{\circ} \mathrm{C}\right)$ y oscuridad, la eficiencia del PSII y la apariencia de las hojas no resultaron afectadas, como cuando las plantas se expusieron a temperatura baja e iluminación $\left(120 \mu \mathrm{mol} \cdot \mathrm{m}^{-2} \cdot \mathrm{s}^{-1}\right)$, condiciones que fueron motivo de PI severa, e incluso aparecieron zonas necróticas en las hojas (Hogewoning y Harbinson, 2007).

Las características de la fotoinhibición dependen básicamente de la cantidad de luz a que las plantas están expuestas. Cuando el exceso de luz es moderado, se reduce la utilización de los quanta pero no se modifica la rata fotosintética máxima. La utilización de los quanta puede volver a tener un valor elevado cuando el flujo de fotones se reduce y queda por debajo del punto de saturación. Este tipo de PI se conoce como fotoinhibición dinámica y se origina mediante la conversión en calor de la energía lumínica absorbida (Osmond, 1994).

Por otro lado, cuando el exceso de luz es extremo, se pueden causar daños en los fotosistemas y esos daños conducen a un descenso tanto de la utilización de los quanta, como de la rata fotosintética. Este efecto permanece durante un periodo relativamente prolongado (semanas o incluso meses), al contrario de cuando se presenta el fenómeno de fotoinhibición dinámica. Este tipo de PI se conoce como fotoinhibición crónica (Osmond,1994).

En un principio se pensó que la reducción en la utilización de los quanta era el resultado de daños causados en el aparato fotosintético. En el momento se ha aceptado que la reducción de la utilización de los quanta, durante un periodo corto de tiempo, probablemente refleja un mecanismo de protección del vegetal contra el exceso de luz incidente, mientras que la PI crónica se origina en una falla o en una sobrecarga en los mecanismos de protección (Taiz y Zeiger, 2000).

La PI dinámica aparece normalmente en horas del medio día, cuando las hojas están expuestas a una gran cantidad de radiación solar y por tanto, se presenta una reducción en la fijación del carbono. Este efecto se acentúa a temperaturas bajas y la PI puede convertirse en crónica bajo condiciones climáticas extremas. En Brassica napus y en Zea mays, la reducción diaria en la tasa fotosintética como consecuencia de la PI reduce en $10 \%$ la producción de biomasa al final del periodo vegetativo (Long et al., 1994). Este valor parece no ser muy significativo; sin embargo, podría influenciar negativamente la posibilidad de supervivencia de algunas poblaciones de plantas, pues bajo condiciones de PI, se acentúa la competencia por el carbono y sobre su reducción, lo cual puede tener consecuencias negativas (Taiz y Zeiger, 2000). Como es el caso de sistemas de producción donde plantas cultivadas y arvenses se desarrollan en conjunto.

Muchas veces, los vegetales se ven confrontados con el problema de que la energía lumínica incidente puede ser mayor que el requerimiento de NADPH y ATP en el metabolismo fotosintético. Este es, por ejemplo, el caso de un exceso de iluminación en plantas, cuando el metabolismo es insuficiente a causa de la temperatura; a temperaturas altas, cuando los estomas están cerrados para disminuir la pérdida de agua, o a temperaturas bajas, cuando el metabolismo es lento por la reducción de la actividad enzimática. Por tanto, la excitación excesiva de los fotosistemas puede conducir a una reducción considerable de los componentes del transporte de electrones en la fotosíntesis (Heldt, 1996). 


\section{DIFERENCIA EN LA FOTOINHIBICIÓN DE LOS FOTOSISTEMAS I Y II}

El PSII ha sido considerado ampliamente como el objetivo principal, cuando se presenta PI, dado que, como ya se mencionó, el PSI es más estable que el PSII bajo una intensidad luminosa eleva$\mathrm{da}$, y porque su inactivación se observa de manera muy eventual in vivo (Havaux y Eyletters, 1991), a pesar de esto, en membranas aisladas de tilacoides, el PSI es tan susceptible al exceso de luz, como el PSII (Tjus, 1995). El primer objetivo de la PI del PSI bajo iluminación elevada en las membranas de los tilacoides son los aceptores terminales de electrones Fx, FA y FB en el centro de reacción (Inoue et al., 1986) (figura 1). También se ha encontrado que el oxígeno es necesario para la inactivación del PSI bajo condiciones de alta iluminación, lo cual sugiere que los daños en este fotosistema son causados por radicales reactivos de oxígeno (ROS) (Tjus y Anderson, 1993).

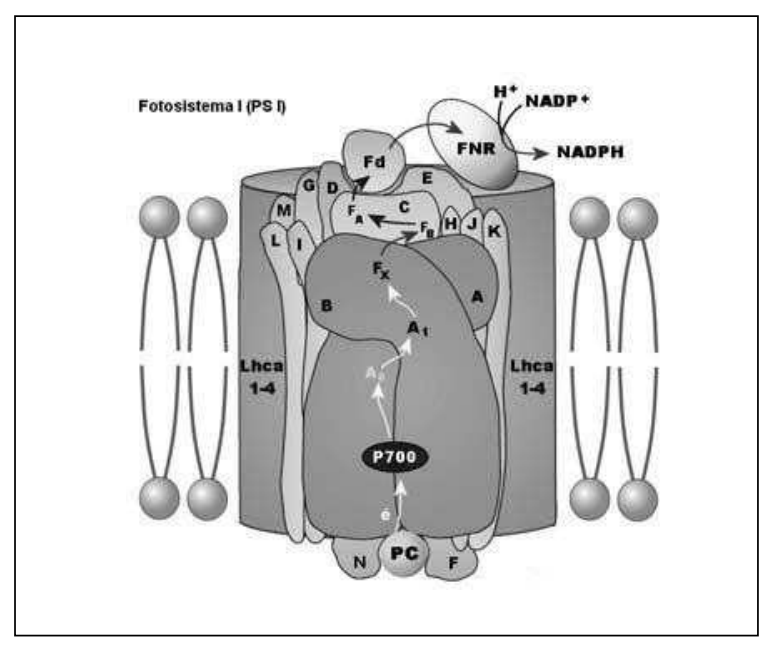

Figura 1. Esquema del fotosistema I (PS I). Tomado de Joly (2004).

La sensibilidad del PSI al exceso de luz en membranas aisladas de tilacoides en comparación con plantas completas demuestra que el complejo del PSI está protegido efectivamente por mecanismos existentes en los cloroplastos, pues el lugar donde se producen los superóxidos en los cloroplastos es cercano al PSI (Asada, 1994). Las enzimas $\mathrm{Cu}$ - y Zn-superóxido dismutasa, que convierten el superóxido en peróxido de hidrógeno, están presentes en concentraciones altas en el estroma del protoplasto, especialmente alrededor del PSI (Ogawa et al., 1995). El peróxido de hidrógeno se reduce a agua mediante la acción de la ascorbato peroxidasa, la cual está unida a los tilacoides del estroma, cerca del PSI (Miyake y Asada, 1992). Por tanto, muy posiblemente, las enzimas reductoras de oxígeno de los cloroplastos podrían servir para proteger el PSI in vivo.

En plantas sensibles a bajas temperaturas, como Cucumis sativus y Solanum tuberosum, los tratamientos con intensidades altas de luz a bajas temperaturas implican que el PSI, más que el PSII, es el principal objetivo celular de la PI in vivo. Se ha propuesto que el daño puede ser causado por oxígeno singlete y por los superóxidos producidos por el PSI; también se ha propuesto que las temperaturas bajas causan una disminución en la tasa de fijación de $\mathrm{CO}_{2}$, lo que tendría como consecuencia, una acumulación del poder reductor en el lado aceptor del PSI, además, se dice que las temperaturas bajas causan lesiones en la doble capa lipídica de las membranas o pueden inactivar las enzimas reductoras de oxígeno, como la superóxido dismutasa. (Havaux y Davaud, 1994; Sonoike y Terashima, 1994; Terashima et al.,1994; Sonoike, 1996; 1996a, b).

En Triticum aestivum se ha encontrado que niveles elevados de radicales activos de oxígeno, como los producidos bajo condiciones severas de estrés, inactivan y degradan la superóxido dismutasa (Casano et al., 1997).

Las condiciones que inducen la fotinhibición del PSI son completamente diferentes a las que permiten este fenómeno en el PSII. En el segundo caso, se requiere una intensidad luminosa muy fuerte, que abarque la completa intensidad de la luz solar; en contraste, la PI del PSI se pue- 
de inducir bajo iluminación débil, a $4-5{ }^{\circ} \mathrm{C}$, por pocas horas. Este fenómeno se puede presentar in vivo, cuando las condiciones de intensidad luminosa son insuficientes para causar PI en el PSI. Además, el hecho de que la PI del PSI no pueda continuar en ausencia de oxígeno, sugiere que en este proceso están involucrados las ROS (Tjus et al., 1998; Scheller y Haldrup, 2005; Sonoike, 1996a).

Se ha propuesto que en el PSII, el oxigeno singlete desarrolla un papel importante en el proceso fotoinhibitorio (Barber y Andersson, 1992), mientras que, de acuerdo con el reporte de Hideg y Wass (1995), no se produce oxígeno singlete en el PSI. Aparentemente, las ROS provenientes del superóxido son bastante importantes en este proceso; además, los fotosistemas I y II responden de manera diferencial a los tratamientos con $\mathrm{H}_{2} \mathrm{O}_{2}$ en condiciones de oscuridad. Está comprobado que la proteína PsaB, componente del centro de reacción del PSI, no se degrada en la oscuridad, mientras que la proteína D1 del PSII sufre alteraciones con este tratamiento. Dado que la magnitud de la PI del PSI está determinada principalmente por la temperatura, mientras que en el PSII tanto la temperatura como la densidad del flujo de fotones determinan este fenómeno, se podría concluir que el PSI es el objetivo cuando se causan daños por bajas temperaturas e intensidad luminosa escasa. En contraste con la proteína $\mathrm{D}_{1}$ del PSII, la cual se restituye rápidamente bajo condiciones de iluminación, la tasa de restitución de la proteína PsaB del PSI no es alta y podría no contribuir al reestablecimiento del fotosistema, luego de la $\mathrm{P}_{\mathrm{I}}$, como sucede con la proteína $\mathrm{D}_{1}$ (Mattoo et al., 1984; Miyao et al., 1995; Jensen et al., 2007) (figura 2).

\section{CONTRIBUCIÓN DE LA RADIACIÓN ULTRAVIOLETA A LA FOTOINHIBICIÓN}

En el trópico, debido al ángulo escaso en el cenit solar y a la delgada capa estratosférica de ozono $\left(\mathrm{O}_{3}\right)$, las plantas soportan niveles de radiación

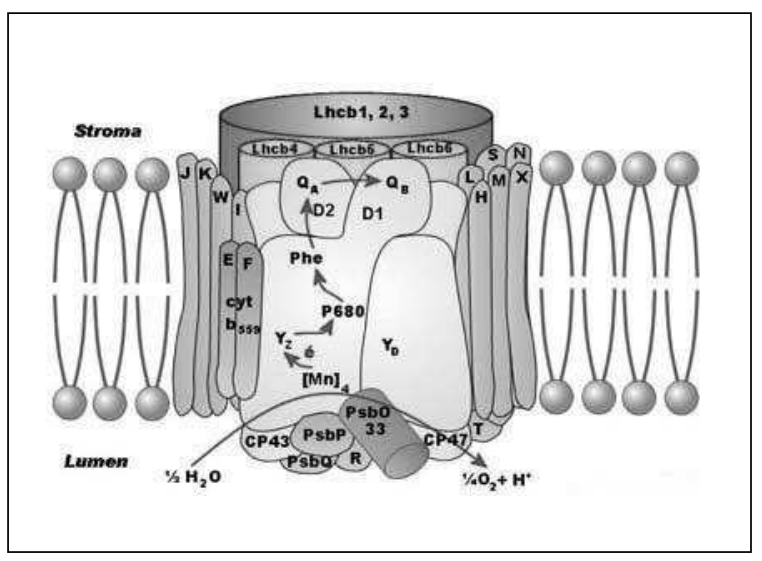

Figura 2. Esquema del fotosistema II (PS II). Tomado de Joly (2004).

UV-B más altos que en otras latitudes. La creciente reducción antrópica de la capa de ozono se ha documentado ampliamente en la región antártica así como la disminución del ozono total en las medias y altas latitudes de ambos hemisferios; sin embargo, la medición de los cambios en los niveles de ozono en el trópico son más problemáticas. Se debe destacar que la radiación UV-B conlleva a daños por fotooxidación, alteraciones en las membranas celulares y en el DNA de los vegetales (Madronich et al., 1995, Knoppik, 1999). Los modelos propuestos por Bojkov y Fioletov (1996) muestran una tendencia negativa en los niveles de $\mathrm{O}_{3}$ en la zona tropical, la cual no es significativa sobre la línea ecuatorial, pero adquieren importancia a partir de $25^{\circ}$ de latitud norte y sur. Aunque esa tendencia puede ser resultante de los eventos naturales recientes, no se puede excluir una futura reducción antrópica de los niveles de $\mathrm{O}_{3}$ en la franja tropical. Algunas evidencias sugieren que las plantas tropicales, a pesar de su alta resistencia propia a la radiación UV-B, pueden resultar afectadas por el incremento de la radiación UV-B, como resultado de la reducción de la capa de $\mathrm{O}_{3}$ (Ziska, 1996). La reducción de la capa de $\mathrm{O}_{3}$ en la franja tropical incrementaría la radiación UV-B incidente sobre las plantas por encima de los niveles máximos soportados por los vegetales en la era geológica actual (Caldwell et al., 1989). 
Las plantas tropicales pueden exhibir una fuerte reducción de la actividad del PSII como respuesta a la radiación UV natural; sin embargo, estas hojas pueden adquirir una protección eficiente contra los rayos UV, dependiendo de la edad de la hoja y del tiempo de exposición a intensidades altas de luz solar. La disminución inducida en el espesor de la capa de ozono permite la entrada de una mayor cantidad de los rayos UV-B, los cuales tendrían un efecto negativo sobre el aparato fotosintético de las plantas tropicales, particularmente en plantas acondicionadas a la luz difusa que de repente se exponen a radiación solar alta (Krause et al., 1998). En las hojas de los árboles tropicales, la radiación UV-A y UV-B puede contribuir a una reducción reversible de la eficiencia potencial del PSII presentada a completa exposición a la luz solar. La sensibilidad del PSII a la radiación UV-B natural depende del grado de acondicionamiento al ecosistema, y del desarrollo de las hojas, y ésta tiende a disminuir con la concentración y la naturaleza de los compuestos vacuolares capaces de absorber los rayos UV. En árboles acondicionados a la sombra, expuestos periódicamente a la luz solar directa, el daño en las proteínas se indicó mediante un efecto fuerte de la radiación fotosintéticamente activa (Photosynthetically active radiation - PAR) y la radiación UV sobre la relación Fluorescencia Variable/Fluorescencia Máxima de la clorofila $\left(\mathrm{F}_{\mathrm{V}} / \mathrm{F}_{\mathrm{M}}\right)$ y sobre la inducción de fluorescencia de la clorofila, así como mediante una restitución extremadamente lenta de la eficiencia potencial del PSII. La reducción en la eficiencia del PSII se disminuyó filtrando los rayos UV-A y UV-B en la luz a la que se expusieron las plantas del ensayo. La fotoinactivación del PSII fue reversible bajo condiciones de sombra, pero la restauración de de casi toda la actividad de este fotosistema requiere por lo menos diez días. En las hojas de árboles acondicionadas a la exposición solar, las cuales contienen niveles altos de compuestos capaces de absorber la radiación UV, se notó un efecto de los rayos UV-B sobre la eficiencia del
PSII, el cual varió con el estado de desarrollo y el grado de aclimatación de las hojas a las condiciones de iluminación (Krause et al., 1999). La radiación natural UV-A y UV-B en la franja tropical, pueden contribuir de manera significativa a que se presente el fenómeno de PI del PSII durante la exposición directa de las hojas a los rayos solares in situ, especialmente en hojas ubicadas a la sombra en la corona de los árboles, cuando se exponen a la totalidad de la radiación solar (Krause et al., 1998; 1999).

\section{MECANISMOS DE PROTECCIÓN CONTRA LA FOTOINHIBICIÓN}

Se ha encontrado que las modificaciones anatómicas desarrolladas por las plantas a través de su evolución y de la presión de selección, han contribuido a su protección contra los efectos negativos del exceso de radiación solar. Es así, como en Digitalis minor la variedad 'Minor' (con tricomas en las hojas) mostró mayor habilidad que la variedad 'Palaui' (desprovista de tricomas) en cuanto a la protección de la maquinaria fotoquímica bajo condiciones ambientales que inducen a la fotoinhibición (Galmez et al., 2007).

La clorofila triplete $\left({ }^{3} \mathrm{Cl}^{*}\right)$, que aparece como subproducto ocasional en la antena y en el centro de reacción a partir de los intermediarios altamente reactivos de las rutas fotosintéticas, es tóxica básicamente porque cede su energía de excitación al oxígeno y genera oxígeno singlete $\left(102^{*}\right)$, que es difusible y tiene la capacidad de reaccionar inespecíficamente con numerosos componentes celulares. Los carotenoides, como el $\beta$-caroteno, tienen la característica de desactivar tanto el estado triplete de la clorofila $\left({ }^{3} \mathrm{Cl}^{*}\right)$ como el singlete del oxígeno $\left({ }^{1} \mathrm{O}_{2}{ }^{*}\right)$. En ambos casos, este compuesto actúa como aceptor de energía de excitación, transformándose en carotenoide triplete $\left({ }^{3} \mathrm{Car}^{*}\right)$ cuya energía ya no alcanza para la formación de oxígeno singlete, así que disipa 
su energía en forma de calor. De esta manera, los carotenoides tienen una función de protección muy importante. Aún se discute, si la función protectora de los carotenoides es insuficiente cuando se presenta una excitación excesiva del PSII, y el oxígeno singlete producido causa daños al complejo PSII, probablemente a nivel de la proteína D1 (Ramírez,1996; Heldt, 1996).

Por otro lado, se han propuesto algunos mecanismos de protección del PSI a la PI, como modo de disipación del exceso de energía lumínica, algunos de ellos son: la llamada Ruta Asada (Asada Pathway), la disociación funcional del LHC (Light harvesting complex) del núcleo del complejo PSII mediante la fosforilación de proteínas, el ciclo de las xantofilas, el flujo cíclico de electrones alrededor del PSI, la regulación baja de la actividad mediante un gradiente de protones creado en las membranas de los tilacoides, y por último, la degradación de la proteína D1 (Yordanov y Veleikova, 2000).

Muy probablemente, el mecanismo de la Ruta Asada es el más importante. Cuando las Ros se reducen en las membranas de los tilacoides o en el estroma, y los electrones fluyen de $\mathrm{H}_{2} \mathrm{O}_{2}$ a $\mathrm{H}_{2} \mathrm{O}$ (Asada, 1994; 1999), el oxígeno acepta electrones del PSI y produce superóxido, el cual es modificado a través de la superóxido dismutasa y la ascorbato peroxidasa hasta convertirse en $\mathrm{H}_{2} \mathrm{O}$. Esta ruta funciona no sólo para disminuir la sobrerreducción del PSI, sino que también genera un gradiente de pH que regula el flujo de electrones del PSII al PSI (Schreiber et al., 1995).

El ciclo de las xantofilas puede ser también de gran importancia en el mecanismo de protección contra la PI, dado que se han encontrado los metabolitos secundarios de este ciclo en una fracción del PSI. El ciclo del flujo de electrones alrededor del PSI puede disipar la energía lumínica absorbida por este fotosistema, pero el flujo de electrones provenientes del PSII no se disminuye lo suficiente; por tanto, la reducción en la acti- vidad del PSII mediante una regulación baja es necesaria para la protección del PSI de la PI (Lee y Thornber, 1995). A pesar de esto, de manera particular en la halofita Artimisia anethifolia, expuesta a condiciones de salinidad, los niveles de zeaxantina y anteraxantina fueron mayores en días claros $\left(1500 \mu \mathrm{mol} \cdot \mathrm{m}^{-1} \cdot \mathrm{s}^{-1}\right)$ que en días nublados $\left(700 \mu \mathrm{mol} \cdot \mathrm{m}^{-1} \cdot \mathrm{s}^{-1}\right)$, evidenciando que ni la fotoinhibición ni el ciclo de las xantofilas se indujeron bajo condiciones de salinidad en esta planta (Lu et al., 2003).

Además de las reacciones bioquímicas inherentes al metabolismo vegetal, bajo condiciones de PI, se han obtenido resultados alentadores con la aplicación exógena de $\mathrm{CaCl} 2$ en Rumex sp., orientada a la reducción del efecto negativo de la PI en condiciones salinas por $\mathrm{NaCl}$, teniendo como referentes la disipación fotoquímica $\left(\mathrm{q}_{\mathrm{p}}\right)$, la eficiencia en la captura de la energía de excitación por parte del PSII $\left(\mathrm{F}_{\mathrm{V}}{ }^{\prime} / \mathrm{F}_{\mathrm{M}}{ }^{\prime}\right)$ y la eficiencia cuántica en el transporte de electrones en el PSII $\left(\varphi_{\text {PSII }}\right)$ (HuaXin et al., 2007). De igual manera, la aplicación exógena de glicinbetaina $\left(100 \mathrm{mM} \cdot \mathrm{L}^{-1}\right)$ en hojas bandera de Triticum aestivum expuestas a estrés hídrico, indujo que las plantas se recuperaran con mayor rapidez del efecto de la PI, que plantas no tratadas (Ma et al., 2006).

En los cloroplastos, la vitamina $\mathrm{E}$ en conjunto con el ciclo de las xantofilas, lleva a cabo dos funciones diferentes en los dos sitios donde tiene lugar la mayor producción de oxigeno singlete: preservando el PSII de la fotoinactivación y protegiendo los lípidos de la membrana de la fotooxidación (Havaux et al., 2005).

\section{CONCLUSIONES}

- Las dimensiones de la PI pueden variar enormemente de acuerdo con el estado fisiológico del vegetal y con las variaciones en el medio ambiente en que éste se desarrolla; es así como la intensidad luminosa y la duración de la ex- 
posición a la luz tienen una gran influencia sobre el fenómeno de PI.

- A pesar de que las condiciones que inducen la fotinhibición del PSI son completamente diferentes a las que permiten este fenómeno en el PSII, la PI en ambos fotosistemas es igualmente importante.

- El hecho de que un exceso de luz tenga como consecuencia un estado de PI, parece depender básicamente de la eficiencia de los mecanismos de protección y de los procesos de recuperación propios de la planta.

- El interés de los científicos con respecto a la PI se ha concentrado especialmente en interpretar los fundamentos bioquímicos de los procesos que conllevan a un estado de PI y que tienen como consecuencia, desórdenes fisiológicos en las plantas.

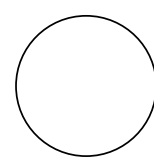

REFERENCIAS BIBLIOGRÁFICAS

Adir, N.; H. Zer; S. Shochate I. Ohad. 2003. Photoinhibition - a historical perspective. Photosynth. Res. 76, 343-370.

Andersson, B. y S. Styring. 1991. Photosystem II: Molecular organsation, function and acclimation. Plant Physiol. Biochem. 31, 683-691.

Aro, E-M.; I.Virgin y B. Andersson. 1993. Photoinhibition of photosystem II. Inactivation, protein damage and turnover. Biochem. Biophys. Acta 1143, 113-134.

Asada, K. 1994. Production and action of active oxigene species on photosynthetic tissues. En: Causes of Photooxidative stress and amelioration of defense systems in plants. Foyer, C.F. y P.M. Mullineaux (eds.). CRC Press, Boca Ratón, Florida. pp. 77-104.

Asada, K. 1999. The water-water cycle in chloroplasts: Scavenging of active oxigens and dissipation of excess photons. Annu. Rev. Plant Physiol. Plant Mol. Biol. 50, 601-639.

Barber, J. y B. Andersson. 1992. Too much of a good thing can be bad for photosynthesis. Trends Biochem. Sci. 17, 61-66.

Bjokov, R.D. y V.E. Fioletov. 1996. Total ozone variations in the tropical belt: An application for quality of ground based measurements. Meteorol. Atmos. Phys. 58, 223-240.

Caldwell, M.M.; A.H. Teramura y M. Tevini. 1989. The changing solarultraviolet climate and the ecological consecuences for higher plants. Trends Ecol. Evol. 4, 363-367.
Casano, L.M.; L.D. Gomez; H.R.Lascano; C.A. Gonzales y V.S. Trippi. 1997. Inactivation and degradation of CuZn-SOD by active oxigen species in wheat chloroplasts exposed to photooxidative stress. Plant Cell Physiol. 38, 433-440.

Demming-Adams, B. y W.W. Adams. 1992. Photoprotection and others responses of plants to high light stress. Annu. Rev. Plant Physiol. Plant. Mol. Biol. 43, 599-626.

Galmés, J.; H. Medrano y J. Flexas. 2007. Photosynthesis and photoinhibition in response to drought in a pubescent (var. minor) and a glabrous (var. palaui) variety of Digitalis minor. Environ. Exp. Bot. 60(1). 105-111.

Havaux, M. y A. Davoud. 1994. Photoinhibition of photosynthesis in chilled potato leaves is not correlated with a loss of photosystem II activity. Preferencial inactivation of photosystem I. Photosynth. Res. 40, 75-92.

Havaux, M. y M. Eyletters. 1991. Is the in vivo photosystem I function resistant to photoinhibition? An answer from photoacoustic and far-red absorbance meassurements in intact leaves. Z. Naturforsch. 46c, 1038-1044.

Havaux, M.; F. Eymery; S. Porfirova; P. Rey y P. Dömann. 2005. Vitamin E protects against photoinhibition and photooxidative stress in Arabidopsis thaliana. Plant Cell 17, 3451-3469. 
Heldt, H. 1996. Pflanzenbiochemie. Spektrum Akademischer Verlag, Heidelberg. pp. 110-112.

Hideg, E. el. Wass. 1995. Singlet oxigen is not produced in photosystem I under photoinhibitory conditions. Photochem. Photobiol. 62, 949-952.

Hogewoning, S.W. y J. Harbinson. 2007. Insights on the development, kinetics, and variation of photoinhibition using chlorophyll fluorescence imaging of a chilled, variegated leaf. J. Exp. Bot. 58(3), 453-463.

Horton, P.; A.V. Ruban y R.G. Walters. 1994. Regulation of light harvesting in green plants. Plant Physiol. $106,415-420$.

Hua-Xin, C.; L. Peng-Min y G. Hui-Yuan. 2007. Alleviation of photoinhibition by calcium supplement in salt-treated Rumex leaves. Physiol. Plan. 129(2), 386-396.

Inoue, K.; H. Sakurai y T. Hiyama. 1986. Photoinactivation of photosystem I in isolated chloroplasts. Plant Cell Physiol. 27, 961-968.

Inoue, K.; Y. Fujii; E. Yokoyama; K. Matsuura; K. Hiyama y H. Sakurai. 1989. The photoinhibition site of photosystem I in isolated choloroplasts under extremely reducing conditions. Plant Cell Physiol. 30, 65-71.

Jensen, P.E.; R. Bassi; E.J. Boekema; J.P. Dekker; S. Jansson; D. Leister; C. Robinson y H.V. Scheller. 2007. Structure, function and regulation of plant photosystem I. Biochim. Biophys. Acta 1767, 335-352.

Joly, D. 2004. Research projects. Université du Québec à Trois-Rivières. En: http://www.uqtr.ca/labcarpentier/eng/research.htm; consulta: julio 2007.

Knoppik, D. 1999. Bedeutung der Photosynthese für die Agrarproduktion und den Kohlenstoffhaushalt der Erde. En: Häder, D.-P. (ed). Photosynthese. Editorial Thieme, Stuttgart. pp. 244-259.

Krause, G.H.; H. Garden; C. Schmude; O.Y. Koroleva y K. Winter. 1998. Contribution of ambient UV light to photoinhibition of photosystem II in tropical plants. En: Garab, G. (ed.). Photosynthesis: Mechanisms and effects. Vol. III. pp. 2409-2412.

Krause, G.H.; C. Schmude; H.Garden; O.Y. Koroleva y K. Winter. 1999. Effects of solar ultraviolet radiation on potential efficiency of photosystem II in leaves of tropical plants. Plant Physiol. 121, 1349-1358.

Lee, A.I.-C. y J.P. Thornber. 1995. Analysis of the pigment stoichiometry of pigment-proetin complexes from barley (Hordeum vulgare). The xanthopyll cycle intermediates occur mainly in the ligth-harvesting complexes of photosystem I and photosystem II. Plant Physiol. 107, 565-574.
Long, S.P.; S.Humphries y P.G. Falkowski. 1994. Photoinhibition of photosynthesis in nature. Annu. Rev. Plant Physiol. Plant Mol. 45, 633-662.

Lu, C.; N. Qiu y Q. Lu. 2003. Photoinhibition and the xanthophyll cycle are not enhanced in the salt-acclimated halophyte Artimisia anethifolia. Physiol. Plant. 118(4), 532-537.

Luciński, R. y G.Jackowski. 2006. The structure, functions and degradation of pigment-binding proteins of photosystem II. Acta Biochim. Pol. 534), 693-708.

Ma, Q.-Q.; W. Wang; Y.-H. Li; D.-Q. Li y Q. Zou. 2006. Alleviation of photoinhibition in drought-stressed wheat (Triticum aestivum) by foliar-applied glycinebetaine. J. Plant Physiol. 163(2), 165-175.

Mattoo, A.K.; H.Hoffman-Falk; J.B.Marder y M. Edelman. 1984. Regulation of protein metabolism: Coupling of photosynthetic electron transport to in vivo degradation of the rapidly metabolized $32-\mathrm{kDa}$ protein of the chloroplast membranes. Proc. Natl. Acad. Sci. $81,1380-1384$.

Madronich, S.; R.L. Mckenzie; M.M. Cadwell y L.O. Björn. 1995. Changes in ultraviolet radiation reaching the earth's surface. Ambio 24,143-152.

Miyake, C. y K. Asada.1992. Thylakoid-bound ascorbate peroxidase in spinach chloroplasts and photoreduction if its primary oxidation product monodehydroascorbate radicals in thylakoids. Plant Cell Physiol. 33, 341-553.

Miyao, M.; M.Ikeuchi; N. Yamamoto y T.A. Ono. 1995. Specific degradation of the D1 of photosystem II by tratment with hydrogen peroxide in darkness: Implication for the mechanism of degradation of the D1 protein under illumination. Biochemistry 34, 10019-10026.

Ogawa, K.; S. Kanematsu; K. Takabe y K. Asada. 1995 Attachment of CuZn-superoxide dismutase to thylakoid membranes at the site of superoxide generation (PSI) in spinach chloroplasts: detection by immunogold labelling after rapid freezing and substitution method. Plant Cell Physiol. 36, 565-573.

Osmond, C.B. 1994. What is photoinhibition? Some insights from comparisions of shade and sun plants. En: Baker, N.R. y J.R. Bowyer (eds.). Photoinhibition of photosynthesis. Oxford (BIOS Scientific). pp. 1-24.

Powels, S.B. 1984. Photoinhibition of photosynthesis in duced by visible light. Annu. Rev. Plant Physiol. 35, 14-44.

Ramirez, J.M. 1996. Fotosíntesis. Absorción y utilización de la luz en la membrana fotosintética. En: AzcónBieto, J. y M. Talón (eds.). Bioquímica y fisiología 
vegetal, McGraw-Hill-Interamericana, Madrid. pp. 91-112.

Scheller, H.V.; A. Haldrup. 2005. Photoinhibition of photosystem I. Planta 221, 5-8.

Schreiber, U.; H. Hormann; K. Asada y C. Neubauer. 1995. $\mathrm{O}_{2}$-dependent electron flow in intact spinich chloroplasts: Properties and possible regulation of the Mehler-Ascorbate-Peroxidase cycle. En: Mathis, P. (ed.). Photosynthesis: From light to biosphere, Vol. II. Kluwer Academic Publishers, Dordrecht. pp. 813-818.

Sonoike, K. 1996a. Degradation of PsaB gene product, the reaction center subunit of photosystem I, is caused during photoinhibition of photosystem I: Possible involvement of active oxigen species. Plant Sci. 115, 157-164.

Sonoike, K. 1996b. Photoinhibition of photosystem I: Its physiological significance in the chilling sensitivity of plants. Plant Cell Physiol. 37, 239-247.

Sonoike, Ke I. Terashima. 1994. Mechanism of photosystem-I photoinhibition in leaves of Cucumis sativa L. Planta 194, 287-293.

Taiz, L. y E. Zeiger. 2000. Physiologie der Pflanzen. SpektrumAkademischerVerlag, Heidelberg. pp.236-237.
Terashima, I.; S. Funayama y K. Sonoike. 1994. The site of photoinhibition in leaves of Cucumis sativa L. at low temperatures is photosystem I, not photosystem II. Planta 193, 300-306.

Tjus, S.E. 1995. Photosystem I, organizational and functional aspects. Tesis de doctorado. Universidad de Estocolmo, Suecia.

Tjus, S.E. y B. Anderson. 1993. Loss of the trans-tylacoid proton gradient is an erarly event during photoinhibitory illumination of chloroplast preparations. Biochim. Biophys. Acta 1183, 315-322.

Tjus, S.E.; B.L. Möller y H.V. Scheller. 1998. Photosystem I is an early target of photoinhibition in barley illuminated at chilling temperatures. Plant Physiol. $116,755-764$.

Yordanov, I. y V. Veleikova. 2000. Photoinhibition of photosystem I. Bulg. J. Plant Physiol. 26(1-2), 70-92.

Zak, E. y H. Pakrasi. 2000. The BtpA protein stabilizies the reaction centre proteins of photosystem I in the Cyanobacterium Synechocystis sp. PCC 6803 at low temperature. Plant Physiol. 123, 223-233.

Ziska, L.H. 1996. The potential sensitivity of tropical plants to increased ultraviolet-B radiation. J. Plant Physiol. 148, 35-41.

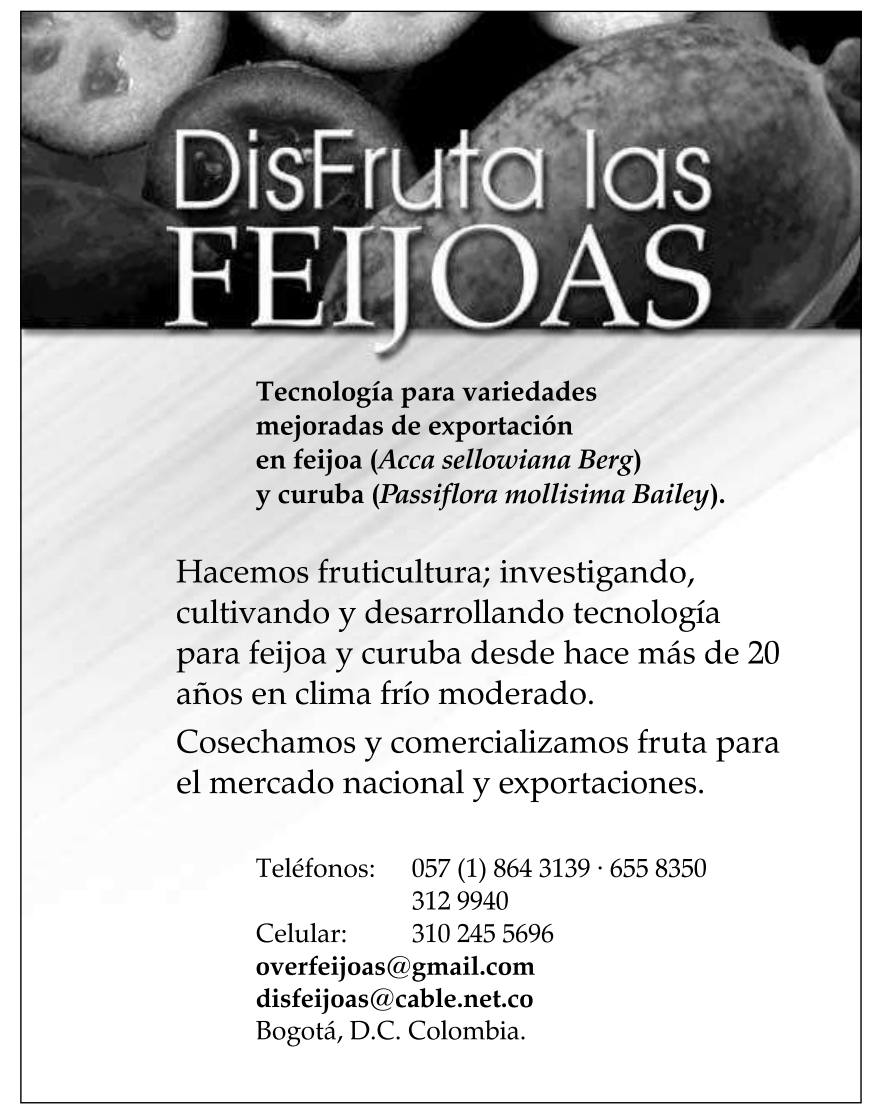

